\title{
Advancements in IR spectroscopic approaches for the determination of fungal derived contaminations in food crops
}

\author{
David McMullin • Boris Mizaikoff • Rudolf Krska
}

Received: 4 July 2014 /Revised: 25 August 2014 / Accepted: 28 August 2014 / Published online: 26 September 2014

(C) The Author(s) 2014. This article is published with open access at Springerlink.com

\begin{abstract}
Infrared spectroscopy is a rapid, nondestructive analytical technique that can be applied to the authentication and characterization of food samples in high throughput. In particular, near infrared spectroscopy is commonly utilized in the food quality control industry to monitor the physical attributes of numerous cereal grains for protein, carbohydrate, and lipid content. IR-based methods require little sample preparation, labor, or technical competence if multivariate data mining techniques are implemented; however, they do require extensive calibration. Economically important crops are infected by fungi that can severely reduce crop yields and quality and, in addition, produce mycotoxins. Owing to the health risks associated with mycotoxins in the food chain, regulatory limits have been set by both national and international institutions for specific mycotoxins and mycotoxin classes. This article discusses the progress and potential of IR-based methods as an alternative to existing chemical methods for the determination of fungal contamination in crops, as well as emerging spectroscopic methods.
\end{abstract}

Keywords IR spectroscopy · Mycotoxin contamination • Chemometrics $\cdot$ Rapid methods $\cdot$ Food chain safety

Published in the topical collection celebrating ABCs 13th Anniversary.

D. McMullin $\cdot$ R. Krska $(\bowtie)$

Center for Analytical Chemistry, Department for Agrobiotechnology,

University of Natural Resources and Applied Life Sciences Vienna,

Konrad-Lorenz-Straße 20, 3430 Tulln, Austria

e-mail: rudolf.krska@boku.ac.at

B. Mizaikoff

Institute of Analytical and Bioanalytical Chemistry, University of

Ulm, Albert-Einstein-Allee 11, 89075 Ulm, Germany

\section{Introduction}

Mycotoxins are widespread, unavoidable natural contaminants of numerous agricultural food and feed commodities produced by filamentous fungi. Mycotoxins enter the food chain by infecting crops that are directly consumed by humans or indirectly as feed ingredients ingested by animals. Fungal contamination may occur pre-harvest in the field or postharvest during storage, and may also be influenced by environmental conditions such as humidity and temperature. These contaminations result in very rapid commodity losses and reductions in quality of economically important commodities such as cereal grains and groundnuts. Trichothecenes, fumonisins, and zearalenone produced by Fusarium species, aflatoxins produced by Aspergillus species, and ochratoxins from Aspergillus and Penicillum species receive the most attention because of their frequent occurrence and deleterious health effects. These toxins are remarkably stable and are not readily degraded or removed by food processing. Mycotoxins elicit a wide range of toxic activities that adversely affect the health of both humans and animals $[1,2]$.

Owing to the human health implications posed by mycotoxins, national and international organizations have adopted regulatory limits for individual mycotoxins and mycotoxin classes that influence international trade. The economic burden of crops contaminated with mycotoxins is additionally increased because of regulatory compliance [3, 4]. Legal requirements to adhere to regulatory limits in agricultural commodities have prompted the development of numerous analytical methods for the determination of mycotoxins. The detection of regulated mycotoxins above the specified legal limit can initiate a series of actions that are costly for industry and, subsequently, the consumer [5]. The analytical requirements at different points of food production chains will vary considerably. A regulator may require more comprehensive instrumentation whereas an easy to use rapid diagnostic test 
may be appropriate for a primary producer. These aspects as well as what defines a rapid test in an industrial setting are discussed in more detail by Miller et al. [5].

Established analytical methods utilized for routine mycotoxin determination include enzyme linked immunosorbent assays (ELISA) [6] and LC-MS/MS [7, 8]. Immunochemical methods such as ELISA rely on antibodies that are typically specific for an individual mycotoxin or class, are relatively quick, easily performed, and are commonly used to screen raw materials [9]. Newer ELISA test kits are commercially available for most of the major mycotoxins at reduced cost per analysis. However, ELISA methods are highly matrixdependent and cross reactivity may result in false positives. Low sensitivity in complex food matrices can also lead to false negatives compared with sophisticated chromatographic methods. Routine reference methods for mycotoxin quantification include HPLC coupled to mass spectrometry, ultraviolet or fluorescence detection or gas chromatography (GC) with electron capture, flame ionization or mass detectors [10]. LCMS/MS based methods offer high sensitivity and selectivity for a wide range of chemically diverse mycotoxins in a single chromatographic analysis. However, many of these methods require time-consuming extraction and cleanup steps. The use of organic solvents is usually not feasible in a non-laboratory setting for employee health and safety reasons. Furthermore, grain elevators maintain a particularly dry environment to prevent mold growth, making the use of water not ideal [5]. Comparatively expensive laboratory procedures requiring technical competence and longer result turnover times increase the financial burden placed upon producers demonstrating the safety of their commodities. These methods generate precise, accurate mycotoxin content data; however, their applicability in the field is not economically practical.

Consequently, new methods are being developed to determine mycotoxin contamination in agricultural commodities, as current strategies do not provide rapid and representative measurements, are costly, time-consuming, and cannot be applied in the field. These new approaches include aptamers [10], optical devices [11], and novel spectroscopic methods. Ideally, a food commodity sample should be analyzed "as is" with minimal sample preparation to be practical within an industrial setting. A more universal approach capable of determining mycotoxin contamination in a variety of solid or liquid raw materials would be more appealing. This could be achieved by determining alterations of inherent food sample properties, such as protein, carbohydrate or lipid content, and texture. Implementing an on-site analytical method that is capable of rapid and reliable determination of fungal contaminants with high accuracy within the imposed upper regulatory limits still remains a challenge. There is a benefit to industry and the consumer from a public health perspective to keep noncompliant food or feed commodities from entering into the food chain because of the adverse health effects caused by mycotoxins.

\section{IR spectroscopic methods for food analysis}

Infrared (IR) spectroscopic methods are among the most promising strategies for determining mycotoxin contamination in agricultural commodities or processed food products. IR-based methods are rapid and nondestructive techniques that require minimal technical training and sample preparation. Analysis is usually not labor-extensive, and large quantities of chemicals are not required in comparison to existing sophisticated chromatographic techniques usually requiring advanced technical competence. These intrinsic qualities of IR-based methods render them an attractive option for high throughput analysis of foodstuffs on site.

The near infrared (NIR) range, $\sim 13,500$ to $4000 \mathrm{~cm}^{-1}$, of the electromagnetic spectrum relies on molecular overtones and vibrations of chemical bonds that can be determined by analyzing reflected or transmitted radiation. The signal intensity depends upon radiation absorption and subsequent alterations for example in the hydrogen bond content within in the matrix. The mid infrared (MIR) range, 4000 to $450 \mathrm{~cm}^{-1}$, relies on molecular vibrations of the matrix constituents. Both the NIR and MIR ranges of the electromagnetic spectrum contain selective information for functional groups (e.g., carbonyl, amide, ester, alcohol, methylene, etc.) present within biomacromolecules (e.g., proteins, lipids, carbohydrates, etc.) found in food commodities. Contaminants including mycotoxins currently cannot be directly determined in a complex matrix such as food because of the limited sensitivity of currently available IR-based methods. Fungal contamination in crops can be indirectly assessed by examining the so-called fingerprint region of the MIR spectrum for alterations in intrinsic crop characteristics. Mycotoxigenic Fusarium species are known to causes alterations in the carbohydrate and protein content, leading to physical deterioration of the crop attributable to fungal and or mycotoxin accumulation. These physical alterations caused by fungal infection can be investigated by examining differences in the spectral bands; 900 $1200 \mathrm{~cm}^{-1}$ for carbohydrates and $1200-1750 \mathrm{~cm}^{-1}$ for protein amide I and II bands; see Fig. 1 [12-14]. However, these spectral differences need to be correlated to reference measurements such as mycotoxin or ergosterol concentrations.

Interpretation of the MIR fingerprint region of complex food matrices remains difficult for discerning a specific component such as carbohydrates, proteins, and lipids. This necessitates the use of chemometrics to extract and correlate IR spectral information to identify distinct spectral feature changes attributable to fungal contamination. Usually, preprocessing of the spectra is required prior to generation of multivariate chemometric models. Qualitative classification methods include principle component analysis (PCA), cluster analysis, artificial neural networks, and K nearest neighbors. Quantitative multivariate models such as principle component regression/principle component analysis (PCR/PCA) and 


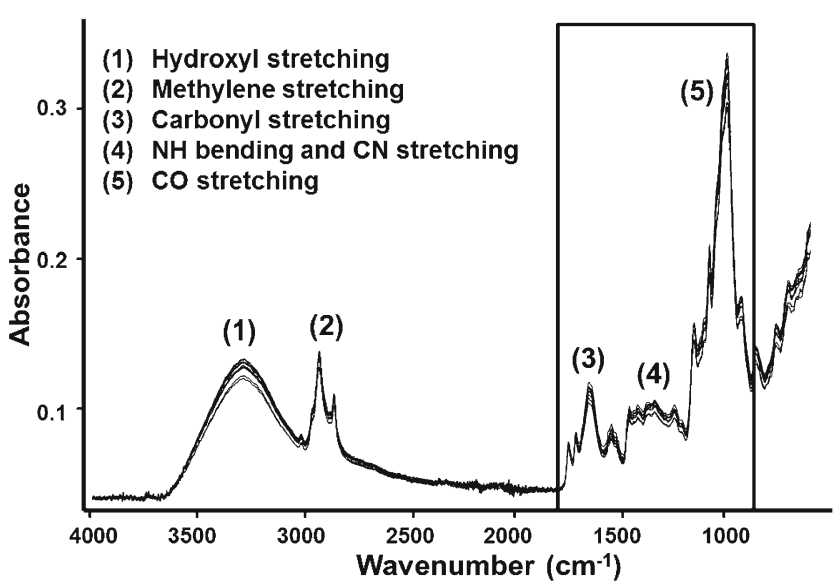

Fig. 1 MIR-ATR spectra $(n=10)$ of sieved $(<250 \mu \mathrm{m})$ maize sample with accompanying band assignments. Within the box, relevant spectral features within the so-called fingerprint region are found

partial least squares regression (PLS) finally enable comparisons of spectra to independent variables such as mycotoxin concentrations. From reviewing the literature, PLS and PCA are among the most frequently utilized chemometric approaches for authentication of food samples. Chemometric techniques commonly utilized for interpretation and analysis of NIR spectroscopic applications have previously been critically reviewed [15]. Multivariate methods determined to be suitable for fungal contamination in specific matrices could then be packaged into easy-to-use software modules for rapid on-site analysis. However, to generate effective calibration models, reliable IR measurements obtained from large sets of samples with accompanying reference measurements (e.g., LC-MS/MS) indicative of fungal contamination are required. Both the quality of the IR spectra and calibration reference method will greatly influence the chemometric model. Finally, it should be emphasized that proper sampling is the basis for reliable analysis. This is vital for both chemometric model generation and on-site analysis of a commodity, as bulk sampling is the largest source of variance [16].

\section{Conventional IR spectroscopy for fungal contamination determination}

IR, particularly NIR spectroscopy is commonly utilized in the food quality control industry to monitor the physical attributes of numerous cereal grains. Information from the NIR region is used in food quality control to measure protein, oil, and starch content [17]. Many reports classify food samples as either healthy or contaminated, whereas some focus on the use of NIR spectroscopy serving as on-line samplers providing spectral feedback for automated sorting of cereal commodities. Most publications focus on NIR spectroscopy, despite MIR offering a higher content of spectral information. MIR has been applied to the detection of microbial spoilage of meat
[18], classification of modified starches [19], apple juice [20], and the determination of fungal contamination in numerous foodstuffs. Pertinent investigations of conventional IR approaches for the determination of fungal contaminations in various food commodities are highlighted here; however, a comprehensive review of the literature on the topic is available [21].

A sample preparation method for increased repeatability of deoxynivalenol (DON) concentration determinations with accompanying IR-attenuated total reflection (ATR) spectroscopy measurements of corn was achieved using the $100-250 \mu \mathrm{m}$ sieve fraction. Sieved samples were classified correctly $100 \%$ of the time as opposed to $79 \%$ for unsieved samples, identifying this as a possibly necessary step for increasing toxin determination accuracy [22]. IR-ATR spectroscopy has also been applied to determining ochratoxin A (OTA) concentrations in contaminated sultans. A concentration range of 2$50 \mu \mathrm{g} / \mathrm{kg}$ was investigated according to the European Union (EU) regulatory limit of $10 \mu \mathrm{g} / \mathrm{kg}$. Spectra of sultanas from various origins showed differences in the fingerprint region and in relation to water content. Reproducibility was increased by standardizing the water activity to 0.62 . Following PCA analysis, samples of $>20 \mu \mathrm{g} / \mathrm{kg}$ could be differentiated from $<10 \mu \mathrm{g} / \mathrm{kg}$ or uncontaminated samples [23].

Transmittance NIR spectra of intact wheat kernels followed by PCA indicated that it may be possible to generate calibration models capable of screening for DON at concentrations just above the EU regulatory limit for wheat flour [24]. In a more recent investigation, 262 wheat samples (143 durum and 119 common) derived from 32 different varieties naturally infected by $F$. graminearum or F. culmorum were analyzed FT-NIR spectroscopy and HPLC for DON quantification. A qualitative model was developed based on PLS analysis facilitated the differentiation of sound and naturally infected samples when setting the DON threshold at $300 \mu \mathrm{g} / \mathrm{kg}$. The model was correct $69 \%$ of the time with 65 validation samples; however, it failed with samples around their set DON threshold (16/20). Hence, the utility of FT-NIR to discriminate DON contamination in unprocessed samples below the set EU regulatory level was demonstrated [13].

Automated sorting of intact wheat kernels was achieved by an NIR spectroscopic method that could differentiate samples at a $60 \mathrm{mg} / \mathrm{kg}$ DON threshold, which was correct $96 \%$ of the time [25]. Similar sorting of corn kernels in a high throughput bimodal fashion has also been demonstrated. Samples were separated into high $(>100 \mu \mathrm{g} / \mathrm{kg})$ and low $(<100 \mu \mathrm{g} / \mathrm{kg})$ aflatoxin groups with over $95 \%$ efficacy [26]. Evaluation of NIR reflectance bands at 715 and $965 \mathrm{~nm}$ could properly identify $98 \%$ of uninfected and $96 \%$ of corn kernels infected by a variety of fungi [27]. These high throughput sorting methods are only applicable in an industrial setting if they are able to discriminate samples near the set regulatory limit for specific mycotoxins within a given commodity. 
NIR accompanied by multivariate statistical analysis is capable of predicting the amount of total fungal infection in maize kernels, as well as the ergosterol and fumonisin B1 concentrations in maize meal [28]. In a more recent investigation, 143 Italian corn meal samples were used for chemometric calibration and external validation was performed with 25 unknown samples. The PLS-based chemometric model developed was able to identify samples with $>4 \mathrm{mg} / \mathrm{kg}$ total fumonisin B1 and B2. This demonstrated the suitability of FTNIR to discriminate contaminated from safe corn meal [29]. NIR spectroscopy was also used to classify maize samples as positive $(>20 \mu \mathrm{g} / \mathrm{kg})$ or negative $(<20 \mu \mathrm{g} / \mathrm{kg})$ for aflatoxin B1 [30]. Both starch content and moisture were shown to greatly affect NIR measurements when detecting aflatoxigenic fungi on rice leading to a low prediction coefficient, $r^{2}=0.68$ [31]. Aflatoxin content was estimated in red chili powder by FT-NIR spectroscopy in diffuse reflectance mode combined with PLS regression analysis. Although high prediction accuracy was achieved between 15 and $500 \mu \mathrm{g} / \mathrm{kg}$, it should be noted that these samples were spiked and not naturally infected or inoculated [32]. FT-IR spectroscopy has also been applied to the direct measurement of fungal natural products [33] and the identification of filamentous fungi [34].

Spectroscopic methods have received increased attention as an economically viable analytical tool for classifying or estimating both mycotoxin and fungal contamination in agricultural commodities. Most reports focus on classifying samples as safe or contaminated based upon alterations in the spectra determined by multivariate analysis; however, better estimations of mycotoxin concentrations appear promising. Current NIR and MIR methods are limited by reference measurements, extensive calibration for individual food matrices, and complex chemometric data interpretation. The sensitivity of NIR and MIR techniques are still not adequate for reliable mycotoxin estimation at the regulatory limits set by the EU. The development of more robust calibration models with larger data sets that reduce the variability of measurements and advanced chemometric software will contribute to NIR and MIR spectroscopy becoming a more viable technique for fungal and mycotoxin contamination determinations within agricultural commodities.

\section{NIR hyperspectral imaging}

The inherent limitations of reference measurements for food and feed safety as well as quality control have necessitated the development of new analytical technologies. Robust and flexible spectroscopic methods that are amenable to on-site applications in the field or production chain suited for screening of raw materials and final products increasing throughput are required. For food quality and safety, NIR hyperspectral imaging (HSI) is an emerging tool that enables the characterization of complex matrices. HSI, also known as chemical or spectroscopic imaging, integrates both conventional imaging and spectroscopy to generate spatial and spectral information of an object [35]. NIR HSIs are three-dimensional arrays of $(m \times k \times \lambda)$ where $m$ and $k$ are spatial axes and $\lambda$ represents the spectral information [36]. HSIs are comprised of hundreds of continuous wavebands for each spatial position of the sample analyzed and its corresponding spectrum [37]. This allows the visualization of biochemical constituents of a particular part of a sample, as regions with similar spectral properties will have similar chemical compositions. A typical HSI system is comprised of an objective lens, spectrograph, camera, acquisition system, illuminator, and computer. Usually, a section of the visible-NIR range (400-1000 nm) is selected and the sample is diffusely illuminated with a tungsten-halogen lamp or LED source. Light is reflected off the sample and separated into its component wavelengths by diffraction optics to generate a two-dimensional image in the spectrograph. The sample is moved past the objective lens on a motorized platform where this process is continuously repeated. Adjacent two-dimensional images acquired are stacked to form a three-dimensional HSIs that can be subsequently processed and analyzed by multivariate statistics [35].

Preprocessing of HSIs is performed to remove nonchemical biases from the relevant information prior to chemometric evaluation. This can remove light scattering effects attributable to inhomogeneities at the sample surface. Chemometrics uses mathematics and statistics to select relevant chemical information by analyzing chemical data, generating useful knowledge of a complex chemical system [38]. Developments in chemometric techniques have facilitated the use of spectroscopic methods because of data reductions allowing more rapid analysis, data extraction, manipulations, and interpretations made possible by increased computing capabilities. This is of consequence for NIR HSI as each sample may constitute thousands of spectra generating very large data files, $>50 \mathrm{MB}$ depending on image and spectral resolution, as opposed to the usual average spectra for classic spectroscopic measurements [39]. Chemometric tools used for data classification include PCA, PLS, linear discernment analysis, artificial neural networks, and multi-linear regression. PLS classification has been demonstrated to be the most popular for discriminating HSI data; however, the conventional methods mentioned herein may not always be suitable for HSI data, since they were designed to analyze single spectra. This necessitates the development of specific chemometric strategies to extract spatial information and relate it to physical or chemical characteristics. There is merit in combining NIR HSI with new chemometric tools to increase food chain safety [39].

NIR HSI is currently being implemented in automated food inspection [40], detection of animal proteins in feed [41], characterization of cereals $[42,43]$, identifying impurities in cereals [41], post-harvest analysis of apples [44], and fungi on food commodities $[45,46]$. NIR HSI is quickly maturing into 
a viable nondestructive method for characterizing fungal contamination on economically important cereals. Visible-NIR HSI was utilized to rapidly discriminate between uninfected and inoculated maize kernels using a 400-1000 nm spectral window. Not only could maize kernels be differentiated as uninfected or A. niger and A. flavus contaminated, but samples could be separated $48 \mathrm{~h}$ post-inoculation. This suggests that early detection of mycotoxigenic fungal contamination in maize is possible [47]. Using 10 different LED lights with emission wavelengths between 720 and $940 \mathrm{~nm}$, ground maize samples were imaged. Using a feed-forward neural network, a significant correlation was observed between fumonisin concentrations and reference measurements [48]. Indirect detection of the fumonisin producer $F$. verticilliodes in maize kernels was achieved with up to $86 \%$ accuracy utilizing NIR HSI [46]. HSI of uncontaminated and $F$. verticilliodes infected maize kernels were analyzed between 1000 and $2498 \mathrm{~nm}$ to monitor fungal development within the kernels. Measurements were performed at predetermined intervals and PCA analysis was utilized to determine the degree of infection. Sources of spectral variation caused by infection were observed at 1900 and $2136 \mathrm{~nm}$, indicative of alterations in starch and protein content [42]. The same group additionally used NIR HSI to differentiate three different Fusarium species, F. subglutinans, F. proliferatum, and F. verticilliodes, with multivariate image analysis. Changes in the mycelium could be observed as a function of time for each strain and species, which enabled differentiation based on scattering variation that was suspected to be due to protein production differences [49].

Similar experiments have also been performed with wheat. The early detection of FHB was investigated in winter wheat using a 400-1000 nm spectral window followed by PCA. Using HSI, the degree of disease was correctly classified $87 \%$ of the time using the spectral differences in two spectral ranges, 665-675 and 550-560 nm, under laboratory conditions [50]. Eight hundred kernels of Canadian Western Red Spring wheat were segregated as sound, mildly damaged, and severely damaged prior to HSI in the same visible-NIR range.
PCA and linear discriminant analysis models were developed to measure the extent of Fusarium damage. Wheat samples were categorized up to $92 \%$ of the time correctly using six wavelengths: $484,567,684,817,900$, and $950 \mathrm{~nm}$. The model was correct $84 \%$ in a validation set [51].

NIR HSI is an emerging tool that enables the characterization of complex heterogeneous samples, including food and feed. Many studies utilizing this technology have indicated that early detection of fungal contamination before disease symptoms become evident is possible. Owing to the continued emphasis for the development of accurate, rapid, nondestructive analytical technologies, it is very likely that these techniques will increasingly be adopted for food safety and quality assurance.

\section{Outlook}

The MIR spectral region at 3-20 $\mu \mathrm{m}$ provides fundamental vibrational and rotational fingerprint absorptions of organic molecules offering inherent molecular selectivity. This renders the spectral window attractive for optical sensing applications including the analysis of complex biological matrices such as food or feed [52]. An MIR sensing system utilizing waveguide-based optical sampling principles is typically comprised of a radiation source, a waveguide to propagate radiation that frequently serves simultaneously as a transducer and a detector. A major limitation of conventional MIR measurements is the achievable sensitivity because of the low intensity of conventional light sources. Advancements in MIR laser technology and, in particular, quantum cascade laser (QCL) technology have rendered them the most promising radiation source for advanced IR sensing applications. Figure 2 depicts an experimental IR set-up encompassing a QCL, planar waveguide, and detector. QCLs offer compact dimensions, high output power, long lifetime, broad tunability $\left(>200 \mathrm{~cm}^{-1}\right.$ for a single laser), room temperature operation, and access to almost the entire MIR spectral band [52, 53]. QCLs are semiconductor lasers that generate light emission via intersubband
Fig. 2 Experimental IR sensor set up coupling a QCL with a GaAs/AlGaAs strip waveguide chip (liquid $\mathrm{N}_{2}$-cooled mercury cadmium telluride semiconductor detector)
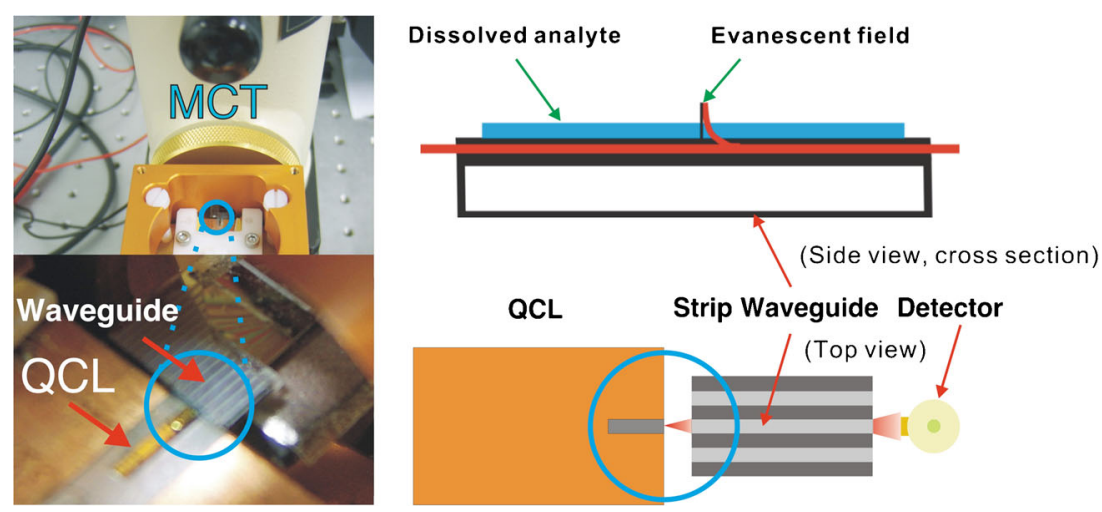
transitions of electrons within the conduction band, taking advantage of a series of quantum heterostructures [54]. In the case of a waveguide-based optical sensor, the transducer is a vital component that translates the chemical or biological signal into an optical signal that may be further processed. Here, it is the waveguide-analyte interface where photons interact with molecular sample constituents that determines the reproducibility of the measurements and quality of the analytical signal. Most MIR sensors are based on internal reflection spectroscopy or, more specifically, ATR techniques. ATR spectroscopy in the MIR range is well established and conventionally uses crystalline materials such as zinc selenide, germanium, or silicon. These configurations allow for a defined yet frequently limited number of internal reflections (i.e., interactions) with the sample present at the waveguide surface. Total internal reflection occurs if light at an angle of incidence exceeding the critical angle is reflected at the interface of an optically denser waveguide and an optically thinner surrounding medium (e.g., an analyte matrix present in liquid or solid state at the waveguide surface). Owing to the optical conditions, electromagnetic radiation propagating along the waveguide leaks into the adjacent environment, which is called an evanescent field. The evanescent field exponentially decays into the sample adjacent to the waveguide with a penetration depth largely determined by the wavelength of the radiation (i.e., few micrometers in the MIR regime) [55]. Until recently, waveguide technology in the MIR band was significantly less developed compared with the visible and NIR spectral regions. A new generation of semiconductor GaAs/AlGaAs thin-film IR waveguides grown by metal-organic vaporphase epitaxy (MOPVD) have been developed that are transparent throughout most of the MIR region [56, 57]. In addition, novel planar waveguides comprised of mercurycadmium-telluride (MCT) also transparent through the MIR region have been reported [53]. Both ideally combine with QCL light sources and may be tailored, frequency-matched to the characteristics of the light source, thereby maximizing the photon-sample interaction. Conventional waveguides are typically much thicker than the applied light wavelength meaning only a few total reflections occur within the device, thereby limiting sensitivity. To improve the signal-to-noise ratio, a thin-film frequency-matched waveguide is required to ensure that most of the energy is transported within the evanescent field where, upon interaction with the analyte, the analytical signal is actually generated (i.e., via evanescent field absorption spectroscopy). This has been demonstrated with the newly developed GaAs/AlGaAs as well as the MCT thin-film waveguides, both providing single-mode behavior and illustrated in Fig. 3. Increasing the intensity of the evanescent field directly affects the achievable signal-to-noise ratio, thereby providing potentially more sensitive measurements in the MIR range compared with conventional (i.e., macroscopic) IR-ATR spectroscopies. Coupling QCLs with these novel

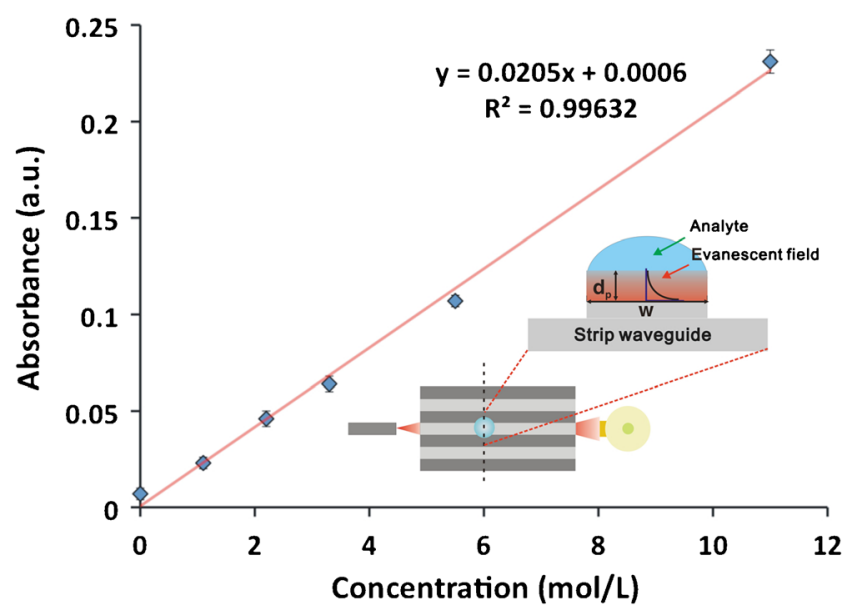

Fig. 3 Sensor response to solutions of acetic anhydride in diethylene glycol mono ether. Each $2 \mathrm{~nL}$ droplet covers an approximate diameter of $0.44 \mathrm{~mm}$ at the strip waveguide surface

thin-film waveguides provides an intriguing opportunity to generate higher quality IR data, which is of particular interest for in-field usage of MIR sensing systems, given the potential of miniaturization by combining thin-film MIR waveguide technology with QCLs. Consequently, it appears that the on-site characterization and analysis capabilities for food or feed samples in a high throughput manner are on the horizon.

Advances in microfabrication and miniaturization of sensor components such as tunable QCLs, detectors and integrated optics, including novel thin-film waveguides, will aid in advancing the applicability of such techniques, particularly in the environment of food and feed analysis. Whereas conventional spectroscopic methods are frequently confined to laboratory settings, combining QCLs with frequency-matched thin-film MIR waveguides has the potential to offer highly sensitive, selective spectral information that can be packaged into miniaturized sensing devices. A currently ongoing research project funded by the European Union (MYCOSPEC; mycospec.eu) is aiming at maturing these technologies toward in-field deployable rapid detection systems. These features would make MIR sensing devices attractive options for the rapid, on-site, determination of fungal contamination in both solid and liquid foodstuffs.

Acknowledgments This study was supported by the European Community's Seventh Framework Programme (FP7/2007-2013), grant number 314018; EU project Mycospec.

Open Access This article is distributed under the terms of the Creative Commons Attribution License which permits any use, distribution, and reproduction in any medium, provided the original author(s) and the source are credited. 


\section{References}

1. Bennett JW, Klich M (2003) Mycotoxins. Clin Microbiol Rev 16: 497-516

2. JECFA (2002) Evaluation of certain mycotoxins in food. (Joint FAO/ WHO expert committee on food additives report 56) WHO Tech Rep 906

3. Dohlman E (2014) Mycotoxin hazards and regulations. Impacts on food and animal feed crop trade. International Trade and Food Safety/ AER-828

4. CAST (2003) Mycotoxins: Risks in plants, animals and human systems. Council for agricultural sciences and technology

5. Miller JD, Schaafsma A, Bhatnagar D, Bondy G, Carbone I, Harris L, Harrison G, Munkvold G, Oswald I, Pestka J, Sharpe L, Sumarah M, Tittlemier S, Zhou T (2014) Mycotoxins that affect the North American agri-food sector: state of the art and directions for the future. World Mycotoxin J 7:63-82

6. Meulenberg EP (2012) Immunochemical methods for ochratoxin A detection: a review. Toxins 4:244-266

7. Berthiller F, Schuhmacher R, Buttinger G, Krska R (2005) Rapid simultaneous determination of major type A- and B-trichothecenes as well as zearalenone in maize by high performance liquid chromatographytandem mass spectrometry. J Chromatogr A 1062:209-216

8. Sulyok M, Berthiller F, Krska R, Schuhmacher R (2006) Development and validation of a liquid chromatography/tandem mass spectrometric method for the determination of 39 mycotoxins in wheat and maize. Rapid Commun Mass Spectrom 20:2649-2659

9. Krska R, Schubert-Ullrich P, Molinelli A, Sulyok M, MacDonald S, Crews C (2008) Mycotoxin analysis: an update. Food Addit Contam 25:152-163

10. McKeague M, Bradley C, Degirolamo A, Visconti A, Miller JD, DeRosa M (2011) Screening and initial binding assessment of fumonisin B1 aptamers. Int J Mol Sci 11:4864-4881

11. Caputo D, De Cesare G, Fanelli C, Manetti C, Nascetti A, Ricelli A, Scipinotti R (2010) Linear photosensor array for on-chip food quality control based on thin layer chromatography. Sensor Lett 8:465-469

12. Hirano S, Okawara N, Narazaki S (1998) Near infrared detection of internally moldy nuts. Biosci Biotechnol Biochem 62:102-107

13. De Girolamo A, Liiolis V, Nordkvist E, Visconti A (2009) Rapid and noninvasive analysis of deoxynivalenol in durum and common wheat by Fourier-transform near infrared (FT-NIR) spectroscopy. Food Addit Contam 26:907-917

14. Peiris K, Pumphrey M, Dowell F (2009) NIR absorbance characteristics of deoxynivalenol and of sound and Fusarium-damaged wheat kernels. J Near Infrared Spectrosc 17:213-221

15. Roggo Y, Chalus P, Maurer L, Lema-Martinez C, Edmond A, Jent N (2007) A review of near infrared spectroscopy and chemometrics in pharmaceutical technologies. J Pharm Biomed Anal 44:683-700

16. Whitaker T, Slate A, Adams J, Birmingham T, Giesbrecht F (2010) Comparing the performance of sampling plans that use a single regulatory limit based upon total aflatoxins to sampling plans that use dual limits based upon B1 and total aflatoxins. World Mycotoxin J 3:35-44

17. Osborne BG (2000) Near-infrared spectroscopy in food analysis. In: Meyers RA (ed) Encyclopedia of Analytical Chemistry. Wiley, Chichester, pp 1-14

18. Ellis D, Broadhurst D, Goodacre R (2004) Rapid and quantitative detection of the microbial spoilage of beef by Fourier transform infrared spectroscopy and machine learning. Anal Chim Acta 514: 193-201

19. Fernández Pierna J, Volery P, Besson R, Baeten V, Dardenne P (2005) Classification of modified starches by Fourier transform infrared spectroscopy using support vector machines. J Agric Food Chem 53:6581-6585

20. Gestal M, Gómez-Carracedo M, Andrade J, Dorado J, Fernández E, Prada D, Pazos A (2004) Classification of apple beverages using artificial neural networks with previous variable selection. Anal Chim Acta 524:225-234

21. Hossain M, Goto T (2014) Near- and mid-infrared spectroscopy as efficient tools for detection of fungal and mycotoxin contamination in agricultural commodities. World Mycotoxin J. doi:10.3920/ WMJ2013.1679

22. Kos G, Lohninger H, Mizaikoff B, Krska R (2007) Optimization of a sample preparation procedure for the screening of fungal infection and assessment of deoxynivalenol content in maize using midinfrared attenuated total reflection spectroscopy. Food Addit Contam 24:721-729

23. Galvis-Sanchez BA, Delgadio I (2007) FTIR-ATR infrared spectroscopy for the detection of ochratoxin A in dried vine fruit. Food Addit Contam 24:1299-1305

24. Pettersson H, Aberg L (2003) Near infrared spectroscopy for the determination of mycotoxins in cereals. Food Control 14:229-232

25. Peiris K, Pumphrey M, Dong Y, Maghirang E, Berzonsky W, Dowell (2010) Near infrared spectroscopic methods for the identification of fusarium head blight damage and prediction of deoxynivalenol in single wheat kernels. Cereal Chem 87:511-517

26. Pearson T, Wicklow D, Maghirang E, Xie F, Dowell F (2001) Detecting aflatoxin in single corn kernels by transmittance and reflectance spectroscopy. Am Soc Agric Eng 44:1247-1254

27. Pearson T, Wicklow D (2006) Detection of corn kernels infected by fungi. Trans Am Soc Agric Eng 49:1235-1245

28. Berado N, Pisacane V, Battilani P, Scandolara A, Pietri A, Marocco A (2005) Rapid detection of kernel rots and mycotoxins in maize by NIR reflectance spectroscopy. J Agric Food Chem 53:8128-8134

29. Gaspardo B, Del Zotto S, Torelli E, Cividino S, Firrao G, Della Riccia G, Stefanon B (2012) Rapid method for detection of fumonisisns B1 and B2 in corn meal using Fourier transform near infrared (FT-NIR) spectroscopy implemented with integrating sphere. Food Chem 135: $1608-1612$

30. Fernandez-Ibanez V, Soldado A, Martinez-Fernandez A, RozaDelgado B (2009) Application of near infrared spectroscopy for rapid detection of aflatoxin B1 in maize and barley as analytical quality assessment. Food Chem 113:629-634

31. Sirisomboon C, Putthang R, Sirisomboon P (2013) Application of near infrared spectroscopy to detect aflatoxigenic fungal contamination in rice. Food Control 33:207-214

32. Tripathi S, Mishra H (2009) A rapid FT-NIR method for estimation of aflatoxin B1 in red chili powder. Food Control 20:840-846

33. Marder L, Corbellini V, Ferrao M, Scroferneker M, Schneider R (2006) Quantitative analysis of total mycotoxins in metabolic extracts of four strains of Bipolaris sorokiniana. Process Biochem 41:17-180

34. Santos C, Fraga M, Kozakiewicz Z, Lima N (2010) Fourier transform infrared as a powerful technique for the identification and characterization of filamentous fungi and yeasts. Res Microbiol 161:168-175

35. Gowen A, O’Donnell C, Cullen P, Downey G, Frias J (2007) Hyperspectral imaging - an emerging process analytical tool for food quality and safety control. Trends Food Sci Tech 18:590-598

36. Williams P, Geladi P, Britz T, Manley M (2012) Investigation of fungal development in maize kernels using NIR hyperspectral imaging and multivariate data analysis. J Cereal Sci 55:272-278

37. Lu R, Chen R (1998) Hyperspectral imaging for safety inspection of food and agricultural products. Proceedings of the SPIE Conference on Pathogen Detection and Remediation for Safe Eating, Boston

38. Massart D, Vandeginste B, Buydens L, De Jong S, Lewi J, Smeyers J (1988) Verbeke, Chemometrics: A Textbook, vol 2. Elsevier, Amsterdam

39. Fernández Pierna J, Vermeulen $P$, Amand $O$, Tossens A, Dardenne $P$, Baeten V (2012) NIR hyperspectral imaging spectroscopy and chemometrics for the detection of undesirable substances in food and feed. Chemometr Intell Lab 117:233-239

40. Qin J (2010) Hyperspectral imaging instruments. In: Da-Wen S (ed) Hyperspectral Imaging for Food Quality Analysis and Control. Elsevier, London, pp 129-172 
41. Fernández Pierna J, Baeten J, Michotte Renier A, Cogdill R, Dardenne P (2004) Combination of support vector machines (SVM) and nearinfrared (NIR) imaging spectroscopy for the detection of meat and bone meal (MBM) in compound feeds. J Chemometr 18:341-349

42. Williams P, Geladi P, Fox G, Manley M (2009) Maize kernel hardness classification by near infrared (NIR) hyperspectral imaging and multivariate data analysis. Anal Chim Acta 653:121-130

43. Manley M, Williams P, Nilsson D, Geladi P (2009) Near infrared hyperspectral imaging for the evaluation of endosperm texture in whole yellow maize (Zeamays L.) kernels. J Agric Food Chem 57:8761-8769

44. Nicolai B, Lotze E, Peirs A, Scheerlinck N, Theron K (2006) Nondestructive measurement of bitter pit in apple fruit using NIR hyperspectral imaging. Postharvest Biol Tec 40:1-6

45. Singh C, Jayas D, Paliwal J, White N (2007) Fungal detection in wheat using near infrared hyperspectral imaging. Trans ASABE 50: 2171-2176

46. Williams P, Manley M, Fox G, Geladi P (2010) Indirect detection of Fusarium verticillioides in maize (Zea mays L.) kernels by near infrared hyperspectral imaging. J Near Infrared Spectrosc 18:49-58

47. Del Fiore A, Reverberi M, Ricelli A, Pinzari F, Serranti S, Fabbri A, Bonifazi G, Fanelli C (2010) Early detection of toxigenic fungi on maize by hyperspectral imaging analysis. Int J Food Microbiol 144:64-71

48. Firrao G, Torelli E, Gobbi E, Raranciuc S, Bianchi G, Locci R (2010) Prediction of milled maize fumonisin contamination by multispectral image analysis. J Cereal Sci 52:327-330

49. Williams P, Geladi P, Britz T, Manley M (2012) Near-infrared (NIR) hyperspectral imaging and multivariate image analysis to study growth characteristics and differences between species and strains of members of the genus Fusarium. Anal Bioanal Chem 404:1759-1769

50. Bauriegel E, Giebel A, Geyer M, Schmidt U, Herppich W (2011) Early detection of Fusarium infection in wheat using hyper-spectral imaging. Comput Electron Agric 75:304-312

51. Shahin M, Symons S (2011) Detection of fusarium damaged kernels in Canada Western Red Spring wheat using visible/near-infrared hyperspectral imaging and principal component analysis. Comput Electron Agric 75:107-112

52. Young C, Kima S, Luzinovaa Y, Weida M, Arnone D, Takeuchi E, Day T, Mizaikoff B (2009) External cavity widely tunable quantum cascade laser based hollow waveguide gas sensors for multianalyte detection. Sensor Actuat B-Chem 140:24-28

53. Wang X, Antoszewski J, Putrino G, Lei W, Faraone L, Mizaikoff B (2013) Mercury-cadmium-telluride waveguides, a novel strategy for on-chip mid-infrared sensors. Anal Chem 85:10648-10652

54. Saleh B, Teich M (2007) Fundamentals of Photonics, vol Chap 17, 2nd edn. Wiley, Hoboken

55. Mizaikoff B (2013) Waveguide-enhanced mid-infrared chem/bio sensors. Chem Soc Rev 42:8683-8699

56. Sieger M, Balluff F, Wang X, Kim SS, Leidner L, Gauglitz G, Mizaikoff B (2013) On-chip integrated mid-infrared GaAs/AlGaAs Mach-Zehnder interferometer. Anal Chem 85:3050-3052

57. Wang X, Kim SS, Roßbach R, Jetter M, Michler P, Mizaikoff B (2012) Ultrasensitive mid-infrared evanescent field sensors combining thin-film strip waveguides with quantum cascade lasers. Analyst $137: 2322-2327$ 